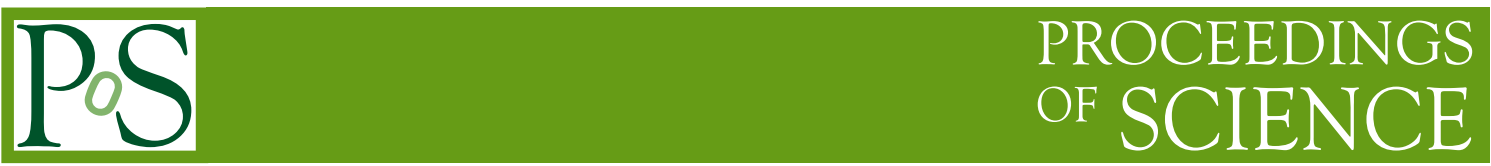

\title{
Gamma-ray Astronomy with DAMPE
}

\section{Shi-Jun Lei ${ }^{* a}$, Qiang Yuan ${ }^{a}$, Zun-Lei Xu ${ }^{a}$, Kai-Kai Duan ${ }^{a b}$, Meng Su ${ }^{c}$ on behalf of the DAMPE collaboration}

${ }^{a}$ Key Laboratory of Dark Matter and Space Astronomy, Purple Mountain Observatory, Chinese Academy of Sciences, Nanjing 210008, China

${ }^{b}$ University of Chinese Academy of Sciences, Beijing, 100012, China

${ }^{c}$ The University of Hong Kong, $H K$

E-mail: sjlei@pmo.ac.cn

Based on 17 months of data from the DArk Matter Particle Explorer (DAMPE), we are able to produce a $\mathrm{GeV}$ gamma-ray sky map showcasing the powerful e/ $\gamma(\mathrm{e} / \mathrm{p})$ discrimination and good angular resolution of DAMPE. Bright gamma-ray point sources of various kinds are identified in the map. We discuss the potential implementation of DAMPE's photon observation in both calibration and science, including boresight alignment, transient sources monitoring, and study of variable sources. Comparison between our result and that of the Fermi is also presented.

35th International Cosmic Ray Conference - ICRC2017

10-20 July, 2017

Bexco, Busan, Korea

\footnotetext{
* Speaker.
} 


\section{Introduction}

The DArk Matter Particle Explorer (DAMPE) is a space-borne high-energy particle detector that measures cosmic rays and gamma-rays in a very wide energy range $[1,2]$ for the study of high energy astrophysics as well as the nature of dark matter particles [3, 4, 5]. As illustrated in Fig. 1, DAMPE is composed of four sub-detectors. The Plastic Scintillation Detector (PSD) acts as anti-coincidence detector (ACD) for gamma-ray identification and a charge detector for the cosmic rays. The Silicon-Tungsten Tracker (STK) accurately measures the track of incident particle. The BGO calorimeter (BGO) measures the energy of the incident particle and provides electron/proton (e/p) separation ability. The Neutron Detector (NUD) provides further e/p separation power $[1,2]$.

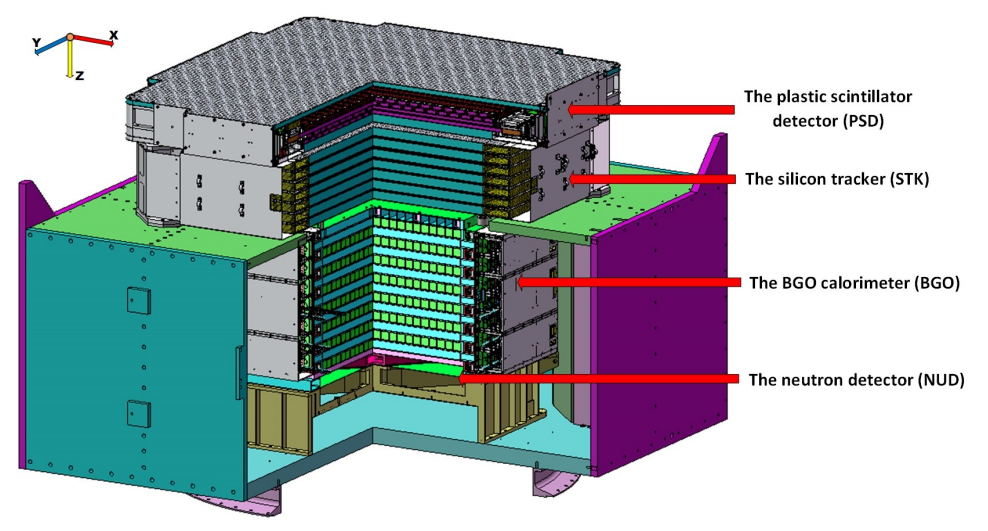

Figure 1: Side view of the DAMPE detector [2].

Gamma-ray observation with high energy, angular, and time resolution is not only helpful for the high energy astrophysics but also for the dark matter indirect detection. A sophisticated photon identification algorithm has been developed for DAMPE to produce a $\mathrm{GeV}$ gamma-ray sky map using 17 months of data. In the following of this article, we first introduce the photon identification algorithm in principle. The validity of the gamma-ray sample, as well as its potential scientific application, is then demonstrated in bright source identification/analysis, transient source monitoring, and boresight alignment.

\section{Gamma-ray selection in DAMPE}

We shown in Fig. 2 the typical detection of a photon (left panel) and a proton (right panel). The PSD on top of DAMPE is used as ACD for gamma-ray identification as the energy deposition of an incident particle in PSD is proportional to the square of the particle charge. An accurate track reconstructed using the STK measurements is then used to determine the PSD bar(s) penetrated by the incident particle and the corresponding path-length. Considering the uncertainties in the measurements of both PSD and STK and the effect of backsplash(s), the PSD is able to provide a rejection power of $\sim 10^{5}$ for charged particles. A first step e/p discrimination with a separation power of $>10^{2}$ is also adapted to enhance the proton suppression. More details about the gamma selection method for DAMPE could be found in [6]. We show in Fig. 3 the acceptance and flux obtained form simulation for photons and electrons, showing that the electron background is 
suppressed to a reasonably level. The same estimation for cosmic protons is not available yet due to limited simulated data, but we are also confident about the proton background given a combined proton rejection power of $>10^{7}$.
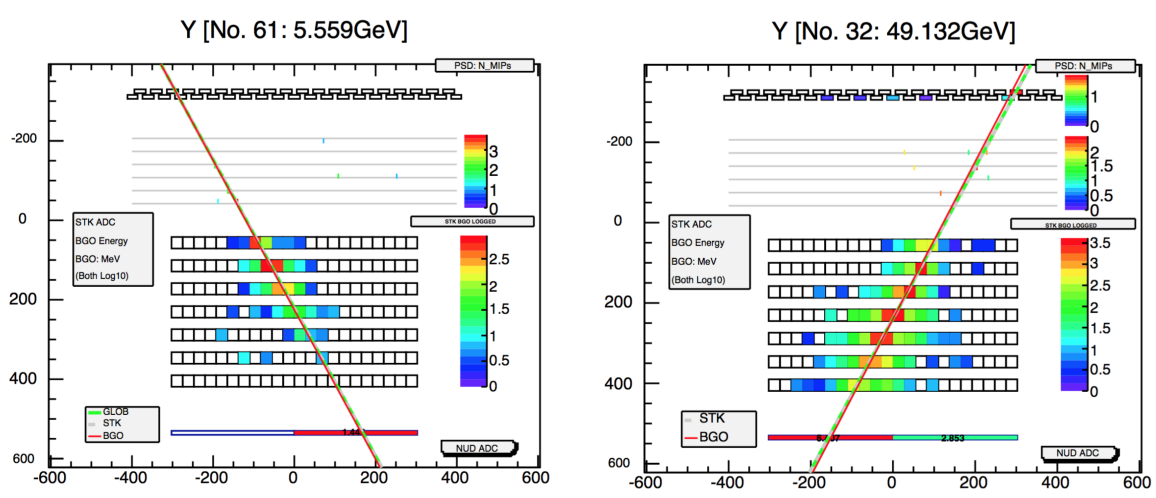

Figure 2: Detection of a typical photon (left) and a proton (right) event. Without igniting the PSD on top of DAMPE, a photon is convert into an electron-positron pair at one of the three tungsten foils equipped in the STK to leave trajectory points in the following STK panels, and then shower electromagnetically in BGO. An incident photon of unit charge ignites the PSD and produce a hardronic shower in BGO after leaving a set of track points in STK.
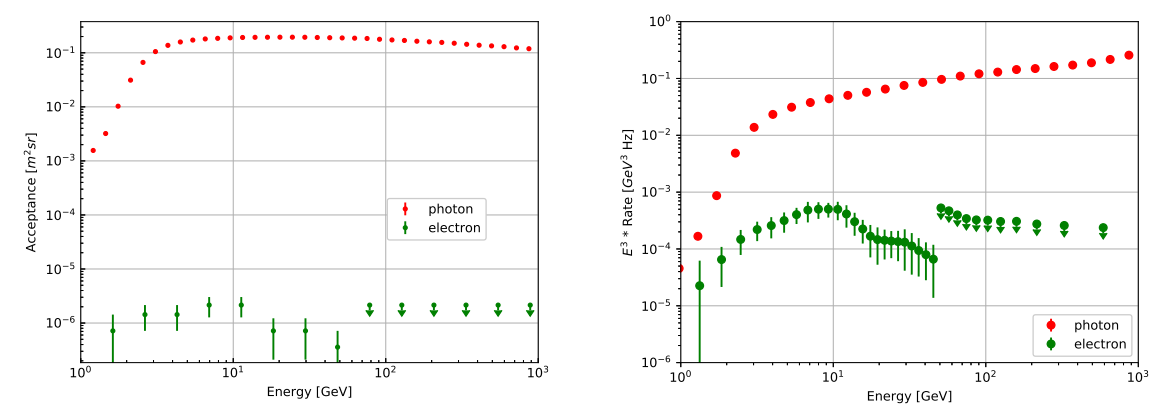

Figure 3: The DAMPE acceptance (left panel) and rate (right panel) of photons and electrons. See [6] for details.

The DAMPE performance in gamma-ray observation is carefully evaluated using simulation data [7]. And a brief summary is given in Tab. 1.

\begin{tabular}{|c|c|}
\hline Parameter Name & Value \\
\hline Energy Range & $5 \mathrm{GeV}-10 \mathrm{TeV}$ \\
\hline Energy resolution at normal incidence & $\sim 1 \% @ 100 \mathrm{GeV}$ \\
\hline Angular resolution at normal incidence & $0.1^{\circ} @ 100 \mathrm{GeV}$ \\
\hline Effective area at normal incidence & $\sim 1200 \mathrm{~cm}^{2} @ 100 \mathrm{GeV}$ \\
\hline Field of View (FoV) & $\sim 1 \mathrm{sr}$ \\
\hline
\end{tabular}

Table 1: Summary of DAMPE expected performance for gamma-ray detection. See [7] for details. 


\section{Bright gamma-ray sources}

Based on the 1.5-year DAMPE observation, we shown in Fig. 4 the GeV gamma-ray sky map without exposure correction. Dozens of sources are clearly identified in the map, including PSR, AGN, SNR etc. A preliminary analysis of some selected bright sources are given in [8]. For example, the spectra and light curves ${ }^{1}$ of the three brightest sources, namely, Vela, Geminga, and Crab are shown in Fig. 5 and 6, respectively.

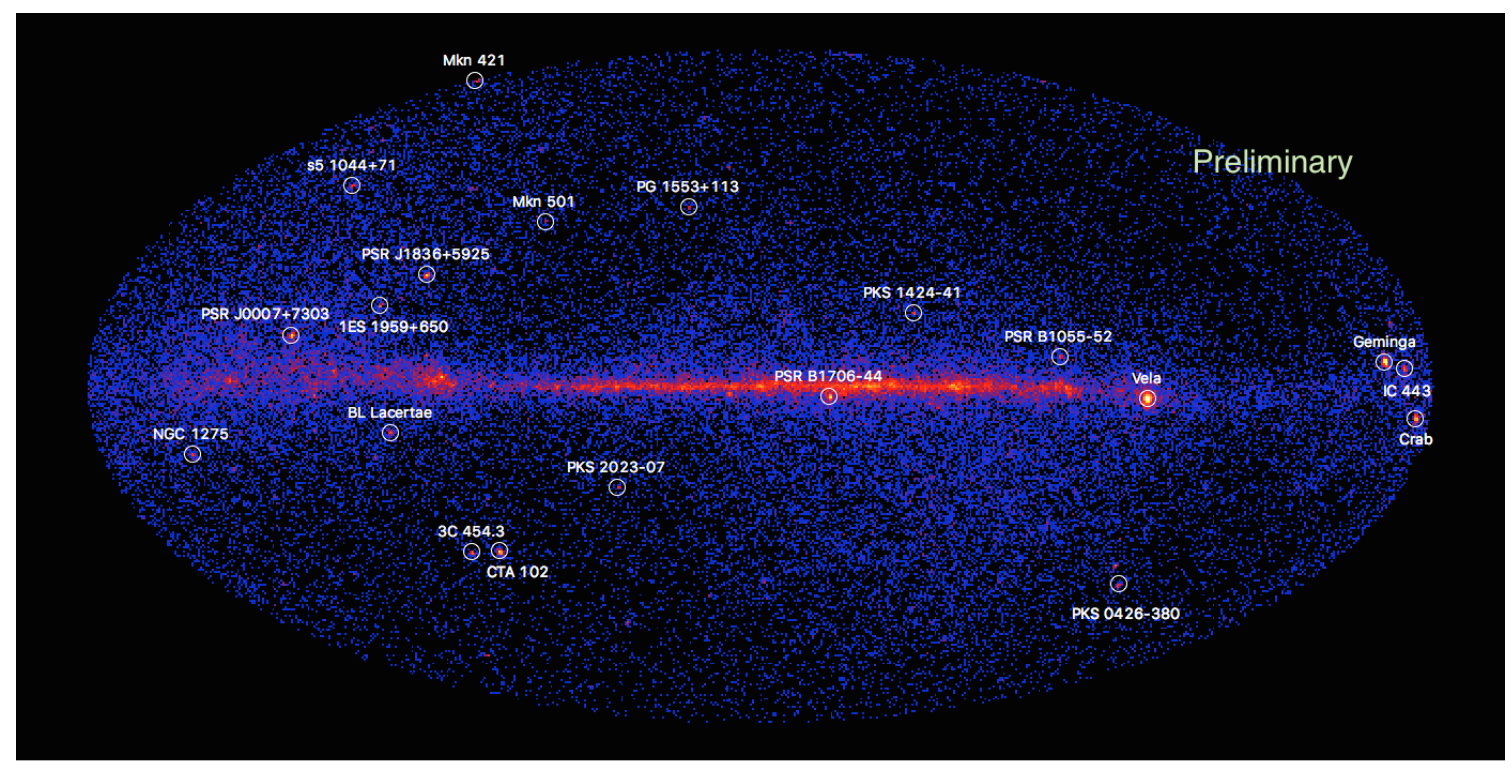

0.3

0.9

2.2

9.5

19.3

155.4

Figure 4: Sources identified in the gamma-ray sky map. See [8] for details.
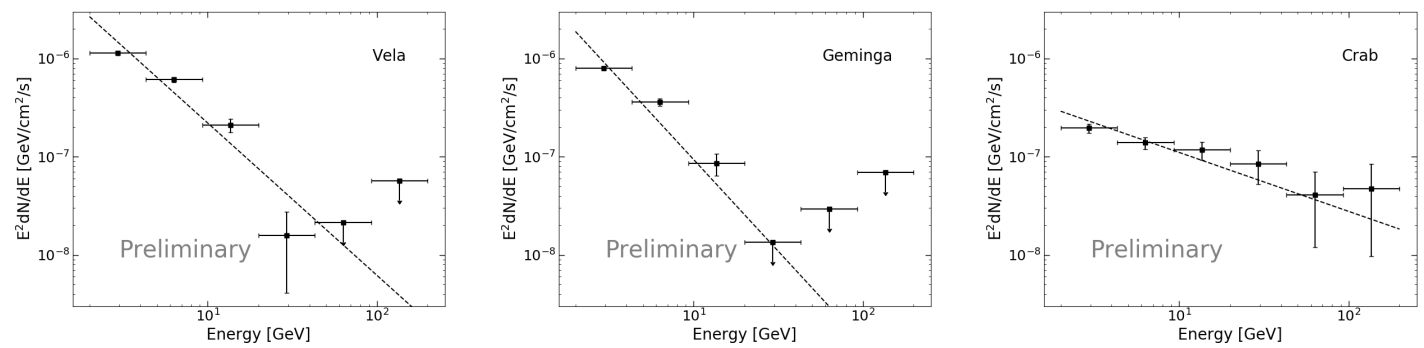

Figure 5: The SED of three bright DAMPE point sources, Vela, Geminga, and Crab. The flux upper limits are calculated if the TS values are smaller than 4. The dashed lines represent the best fitted power law spectrum in the global analysis. See [8] for details.

Using our gamma-ray observation of the famous pulsar Geminga of very good time resolution (up to milisecond), we are able to stack the photons within $3^{\circ}$ of the source to produce the phase

\footnotetext{
${ }^{1}$ A preliminary ephemeris initially from the Fermi-LAT $\gamma$-ray data [?] and further optimized using the Fermi-LAT data observed from 2016-01-01 to 2017-06-01 is used to calculate the light curve.
} 

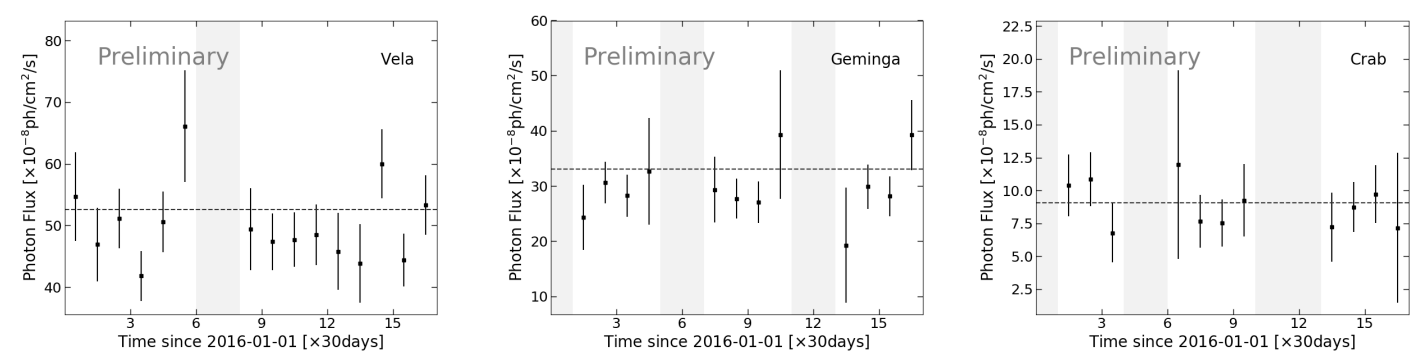

Figure 6: Aperture photometric light curves of Vela, Geminga, and Crab. The time bin size is 30 days. The horizontal dashed line indicates the average flux obtained in global likelihood analysis. The shaded range represents the time bin in which the target source is not within the field of view of DAMPE. See [8] for details.

diagram of the pulsar (red histogram in Fig. 7). A good consistency is seen in comparing our result to that based on the observation of Fermi-LAT (blue histogram in Fig. 7).
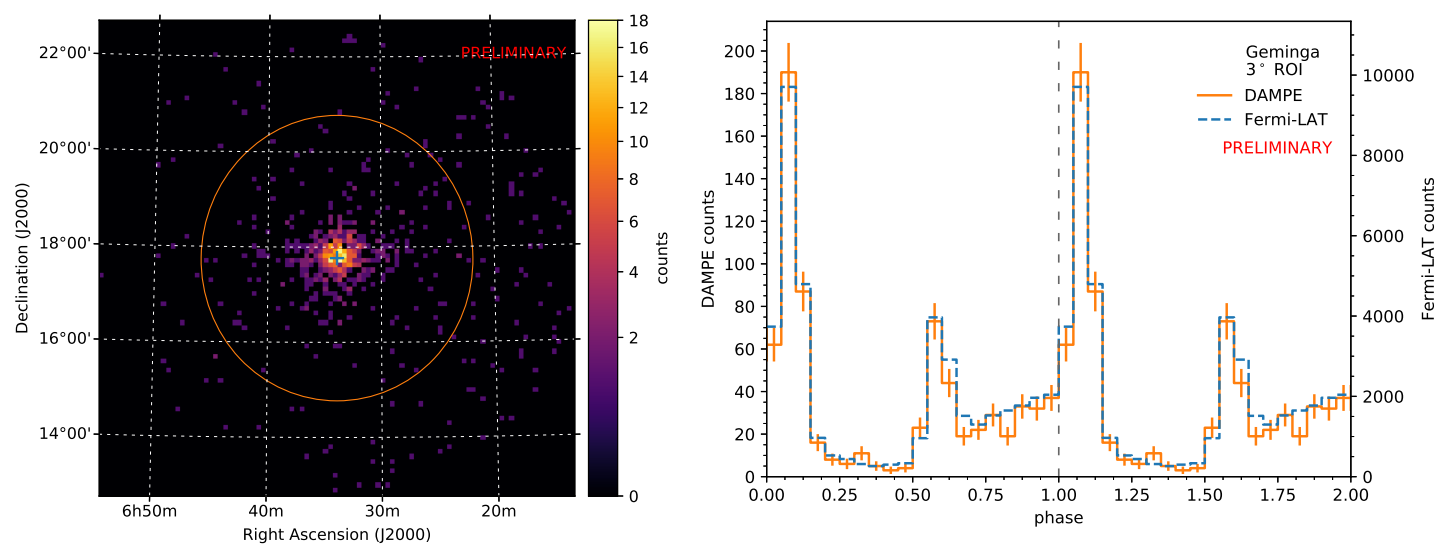

Figure 7: Left panel: $10^{\circ} \times 10^{\circ}$ counts map for all photons above $1 \mathrm{GeV}$ around the Geminga pulsar. The position of the pulsar is indicated by the blue cross. Right panel: The light curve of these photons within $3^{\circ}$ ROI.

\section{Transient source monitoring}

Operating in a solar synchronous orbit with a fairly large field of view, DAMPE monitors more than half of the sky in every revolution of 95 minutes, and covers the entire sky twice annually. Hence the continuous DAMPE gamma-ray observation is suitable for Transient source identification and monitoring. As shown in Fig. 8, a flare is clearly seen in the light curve of CTA 102 based on the first year DAMPE observation.

\section{Boresight alignment}

The direction of each detected particle is reconstructed with respect to the reference system of DAMPE payload. To achieve the celestial coordinate of a particle, the transformation from payload 

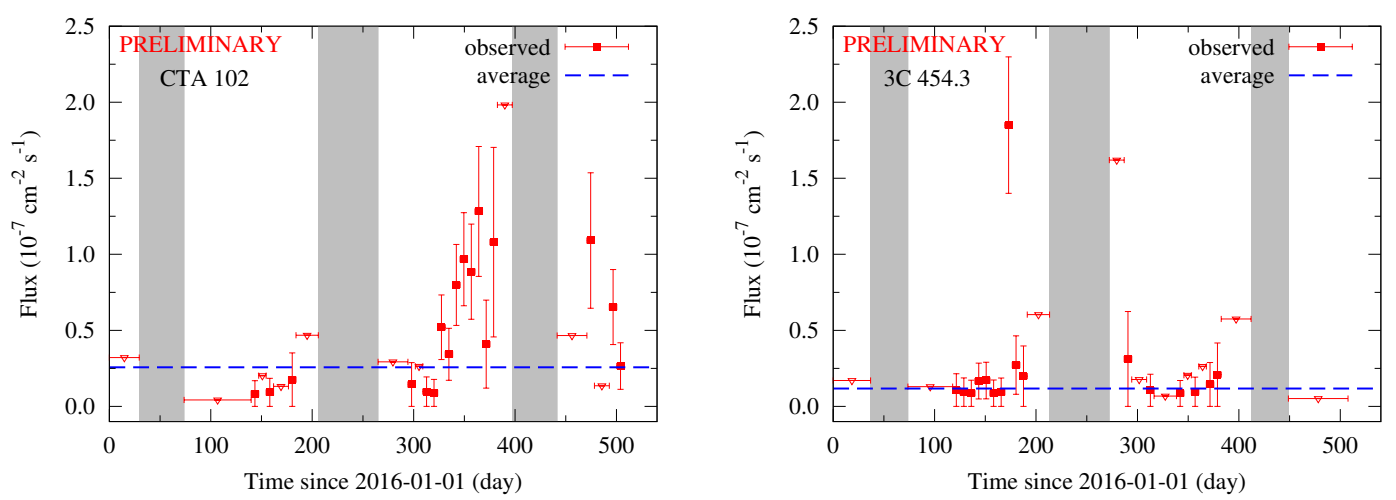

Figure 8: Light curves for the direction of CTA 102 (left panel) and 3C454.3 (right panel). Red squares are the observed flux, and the blue line represents the expected ones assuming steady emission of the source. See [10] for details.

coordinate system to celestial coordinate system is required. The transformation is determined by orbital parameters and the celestial orientation of the satellite which are provided by the Navigation system and star-tracker respectively. Small deviations from real pointing may be introduced to the transformed celestial coordinate, due to thermal variations, acoustic vibrations, $0 \mathrm{~g}$ fluctuations, and uncertainty in the orbital parameters and star-tracker pointing. This miss match will not only cause a systematic shift between the mean observed position and the real position of a point source, but also lead to a distorted point spread function (PSF) profile. In this section, we use the gamma-ray data centered around several bright point sources to measure and correct the angular deviation from the real celestial coordinate, called "boresight alignment" of DAMPE payload.

For this purpose, we use three of the brightest sources, namely, Vela, Geminga, and Crab in the gamma-ray sky, and select photons within $4^{\circ}$ of the real position of the sources and restrict the energy between $3 \mathrm{GeV}$ and $100 \mathrm{GeV}$. The gamma-rays in a region of interest (ROI) can be modeled by a point source and a background template. The spectral and spatial parameters of the point source is import from the third Fermi-LAT catalog of high-energy sources [11]. The background is modeled by an isotropic template with a power-law spectrum.

As summarized in table 1, we see a reasonably good consistency between the results of the boresight alignment using the three different sources.

\begin{tabular}{|c|c|c|c|c|}
\hline Source Name & Photon Number & $\theta_{X}$ (degree) & $\theta_{Y}$ (degree) & $\theta_{Z}($ degree $)$ \\
\hline Vela & 1438 & $0.13 \pm 0.01$ & $0.02 \pm 0.01$ & $-0.14 \pm 0.02$ \\
\hline Geminga & 446 & $0.13 \pm 0.02$ & $-0.02 \pm 0.02$ & $-0.14 \pm 0.02$ \\
\hline Crab & 265 & $0.11 \pm 0.02$ & $-0.03 \pm 0.03$ & $-0.15 \pm 0.03$ \\
\hline
\end{tabular}

Table 2: Boresight alignment results estimated using three brightest gamma-ray sources.

\section{Summary}

DAMPE has been operating smoothly on-orbit for more than one year and a half and more than 2.8 billion cosmic ray and gamma-ray events covering a very wide energy range have been 
recorded. Based on the data and a sophisticated photon identification algorithm, we are able to produce a gamma-ray sky map of more than 90000 photons with low contamination level. We discuss the potential implementation of DAMPE's photon observation in both calibration and science, including identification/analysis of a number of brightest sources, transient sources monitoring, and boresight alignment. Independent researches related to the DAMPE gamma-ray detection are also carried and will be posted elsewhere, including the gamma-ray selection method[12], simulation on gamma-ray detection [13], and analysis of a few bright pulsars based on independent gamma-ray selection method[14].

\section{Acknowladgement}

The DAMPE mission is funded by the strategic priority science and technology projects in space science of Chinese Academy of Sciences. In China the data analysis was supported in part by the National Key Research and Development Program of China (No. 2016YFA0400200), National Basic Research Program of China (No. 2013CB837000), NSFC (Nos. 11525313 and 11622327), the 100 Talents Program of Chinese Academy of Sciences, the Astronomy Joint Grant (U1631111), and Strategic Priority Research Program of the CAS "Multi-waveband Gravitational Wave Universe" (Grant No. XDB23040000). In Europe the activities and the data analysis are supported by the Swiss National Science Foundation (SNSF), Switzerland; the National Institute for Nuclear Physics (INFN), Italy.

\section{References}

[1] J. Chang, Dark Matter Particle Explorer: The First Chinese Cosmic Ray and Hard gamma-ray Detector in Space, Chin. J. Spac. Sci., 34550 (2014).

[2] J. Chang et al. [DAMPE collaboration], The dark matter particle explorer mission, submitted to Asropart. Phys., (arXiv:1706.08453) (2017).

[3] Y. Z. Fan, B. Zhang, \& J. Chang, Electron/positron Excesses in the Cosmic Ray Spectrum and Possible Interpretations, Int. J. Mod. Phys. D, 192011 (2010).

[4] J. L. Feng, Dark Matter Candidates from Particle Physics and Methods of Detection, Annu. Rev. Astron. Astrophys. 48, 495 (2010).

[5] Y. F. Liang, et al., Search for a gamma-ray line feature from a group of nearby galaxy clusters with Fermi LAT Pass 8 data, Phys. Rev. D, 93 (2016) 103525.

[6] Z. L. Xu, et al., Gamma-ray selection of DAMPE, proceeding of ICRC2017 (2017)

[7] K. K. Duan et al, The performance of DAMPE for gamma-ray detection, proceeding of ICRC2017 (2017)

[8] Y. F. Liang et al., Bright gamma-ray sources observed by DArk Matter Particle Explorer, proceeding of ICRC2017 (2017)

[9] Z. L. Xu, et al. DAMPE detection of variable GeV gamma-ray emission from blazar CTA 102, The Astronomer's Telegram No.9901 (2016)

[10] Q. Yuan et al., The variable sky of DAMPE, proceeding of ICRC2017 (2017) 
[11] M. Ajello, et al. [Fermi-LAT collaboration], 3FHL: The Third Catalog of Hard Fermi-LAT Sources, arXiv:1303.3514 (2017).

[12] S. Garrappa et al., A Machine Learning classifier for photon selection with the DAMPE detector, proceeding of ICRC2017 (2017)

[13] M. N. Mazziotta et al., A fast photon simulation algorithm for the DAMPE experiment, proceeding of ICRC2017 (2017)

[14] M. M. Salinas, First observations of Pulsars with the DArkMatter Particle Explorer, proceeding of ICRC2017 (2017) 